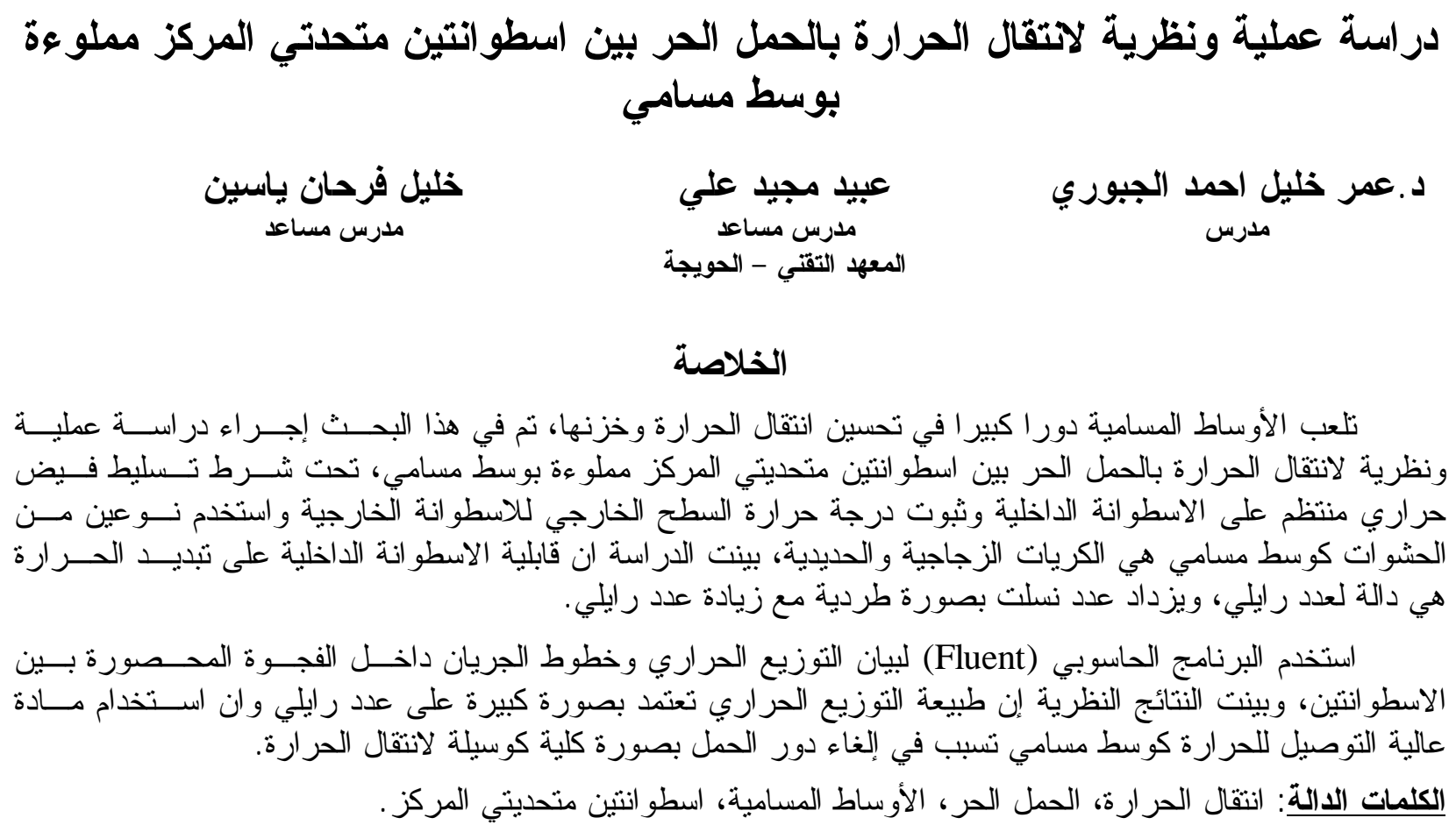

الخلاصة

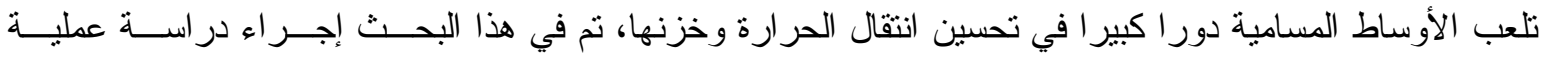

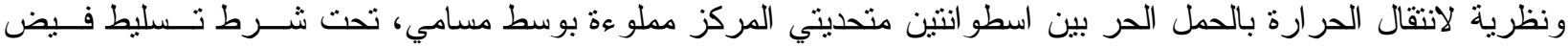

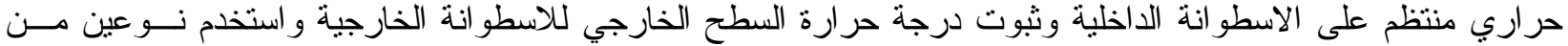

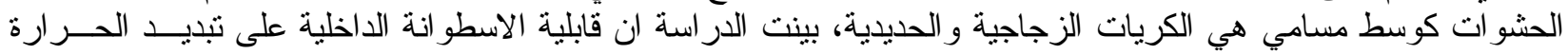

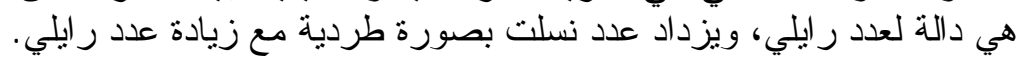

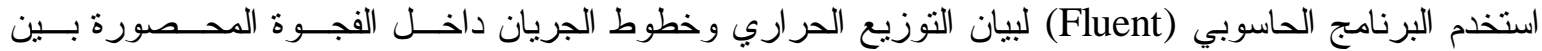

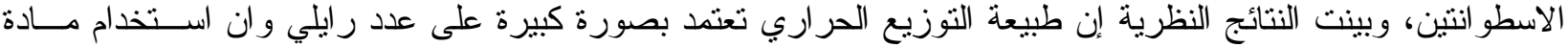

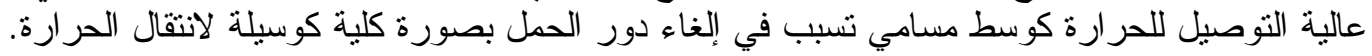
الكلمات الدالة: انتقال الحر ارة، الحمل الحر ، الأوساط المسامية، اسطو انتين متحديتي المركز .

\title{
Expermintal And Theoretical Study Of Natural Convection Heat Transfer Between Two Concentric Cylinders Filled With Porous Medium.
}

\author{
Omar K. Ahmed Al_Joboury Obeed Majeed Ali Khalil Farhan Yaseen \\ Lecturer \\ Asst. Lecturer \\ Asst. Lecturer
}

Technical Institute of Haweja

\begin{abstract}
Porous media have considerable importance in improvement of heat transfer and storage. This research includes an experimental and theoretical study of natural convection heat transfer between two concentric cylinders filled with a porous medium, under condition of applying uniform heat flux on the inner cylinder and constant outer surface temperature for the outer cylinder. Two types of filling material were used as porous medium, iron and glass beads, the study shows that the heat dissipated ability in the inner cylinder is function of Rayligh number, and the Nusselt number increase is directly proportional with the increase in Rayligh number .

Fluent program was used to show the heat dissipation and the flow lines inside the gap between the two cylinders, the experimental results show that the nature of the heat distribution depends greatly on Rayligh number, and the use of highly heat conductive materials as porous medium will completely cancel the convection as a mean of heat transfer.
\end{abstract}

Key wards: Heat transfer, Natural convection, Porous medium, and two concentric cylinders. 


\begin{tabular}{|c|c|c|}
\hline الوحدات & المعنحى & اليرمز \\
\hline- & ثابت القصور & $C_{2}$ \\
\hline $\mathrm{m}$ & قطر الكريات الزجاجية او المعدنية & $d_{p}$ \\
\hline $\mathrm{W} / \mathrm{m} .{ }^{\circ} \mathrm{C}$ & معامل التوصيل الحراري للمائع & $k_{f}$ \\
\hline $\mathrm{W} / \mathrm{m} .{ }^{\circ} \mathrm{C}$ & معامل التوصيل الحر اري للمعدن & $k_{s}$ \\
\hline- & عدد رايلي & $R a$ \\
\hline- & حد المصدر & $S$ \\
\hline${ }^{\circ} \mathrm{C}$ & درجة الحرارة & $\mathrm{T}$ \\
\hline $\mathrm{m} / \mathrm{sec}$ & السر عة باتجاه X & $\mathrm{u}$ \\
\hline $\mathrm{m} / \mathrm{sec}$ & السر عة باتجاه y & $\mathrm{v}$ \\
\hline $\mathrm{m} / \mathrm{sec}$ & السر عة باتجاه Z & $\mathrm{W}$ \\
\hline- & الخاصية & $\phi$ \\
\hline $1 / \mathrm{K}$ & معامل التمدد الحراري & $\beta$ \\
\hline $\mathrm{Kg} / \mathrm{m}^{3}$ & الكثافة الكتلية & $\rho$ \\
\hline- & معامل الانتشار & $\Gamma$ \\
\hline$=$ & مرجعية & $\mathrm{O}$ \\
\hline$\overline{-}$ & المسامية & $\varepsilon$ \\
\hline
\end{tabular}

\section{المقدمة ومر اجعة المصادر:}

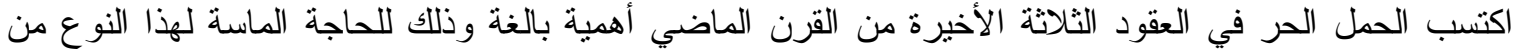

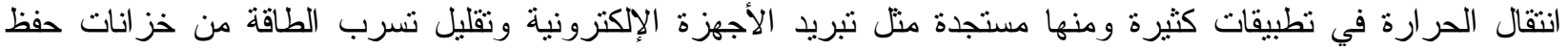

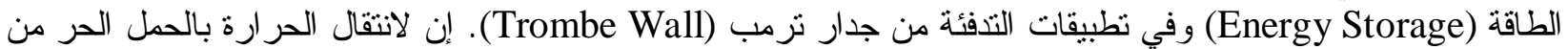

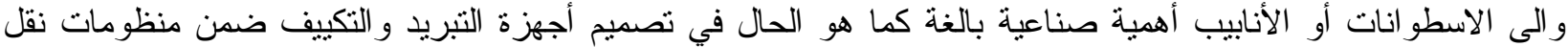

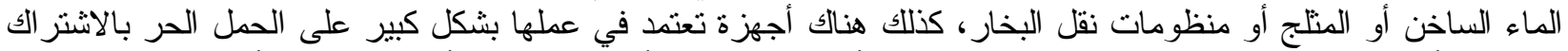

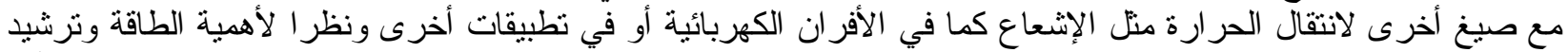

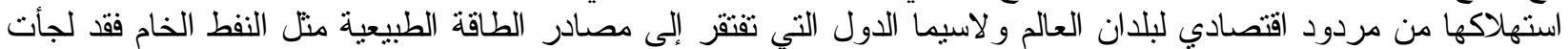

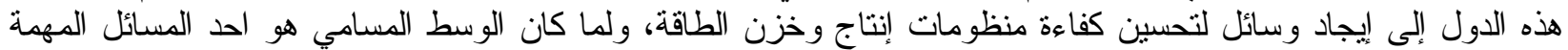

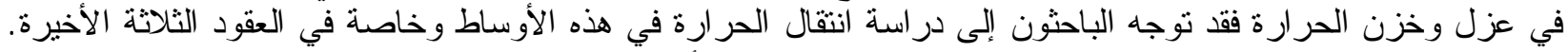
وقد قام الباحثون باستقاء نظري وتجريبي لانتقال الحرارة خلال الأوساط المسامية وللعديد من الإشكال الهندسية وبظروف

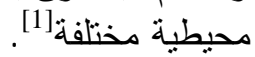

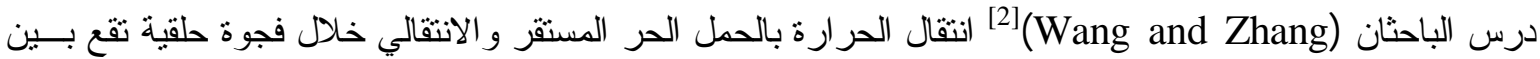

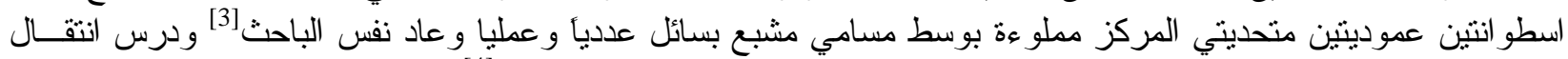

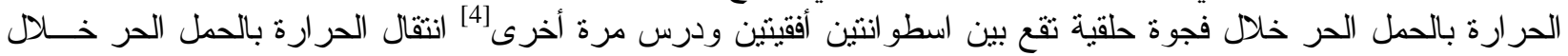

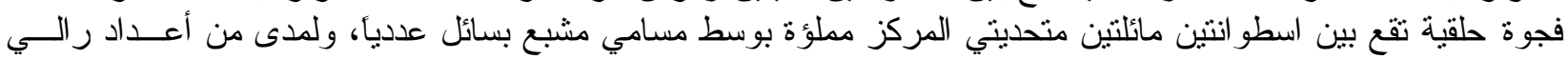

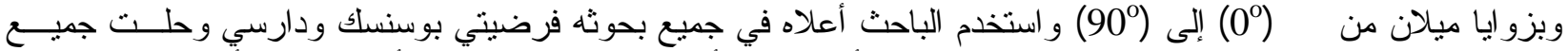

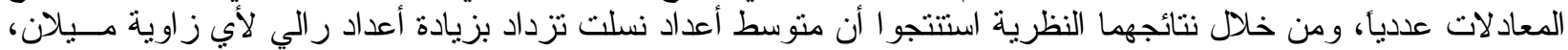

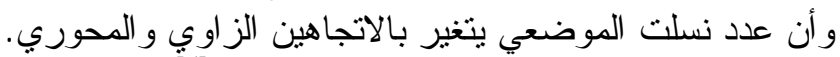

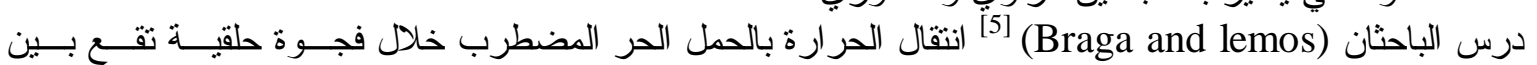
اسطو انتين أفقيتين متحديتي المركز مملؤة بوسط مسامي بصورة نظرية وباستخدام نموذجين من نماذج الجريان المــضطرب 
الخمسة، وتوصل الباحث إلى مجموعة من العلاقات الارتباطية التي تحكم انتقال الحرارة في الـشكل الهندسـي المـستخدم

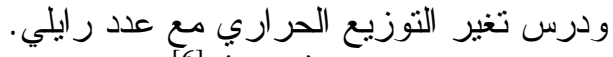

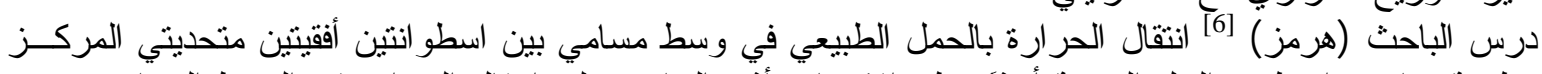

بصورة نظرية وباستخدام طرق الحل العددية أخذا بنظر الاعنبار تأثثر الحاجز على انتقال التقال الحرارة في الوسط المسامي .

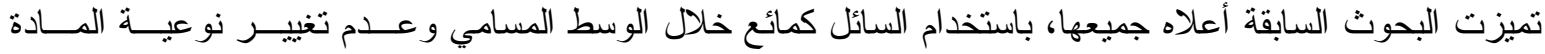

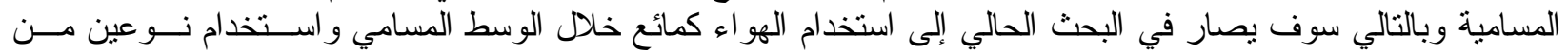

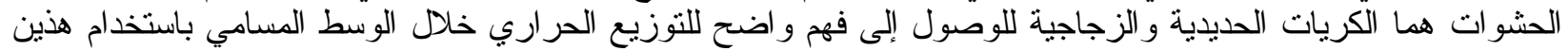
النوعين من الحشوات وللوصول إلى علاقات ارتباطية تحدد معدل انتقال الحرارة بالحمل الحر فيها و لإعداد نسلت و اطئة.

تضمن الجانب العملي دراسة انتقال الحر ارة بالحمل الحر داخل الفر اغ الحلقي المحصور بين اسطو انتين متحديتي

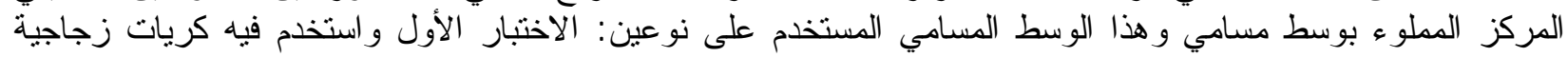
بقطر 11 mm و غير إلى كريات حديدية بقطر 3.28 mm في الاختبار الثاني، بني لهذا الغرض منشأ تجريبي (الثنكل (1)) يتألف أساسا من عنصر التسخين (الاسطوانة الداخلية) وهو عبارة عن اسطوانة من الألمنيوم بقطر خارجي الإني (22 mm)

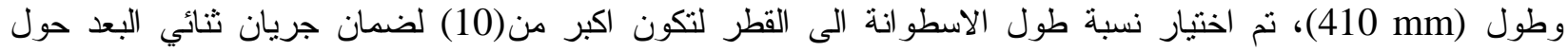

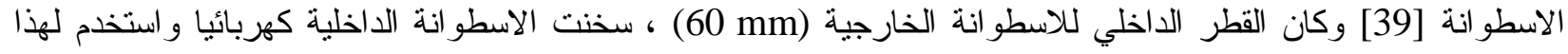

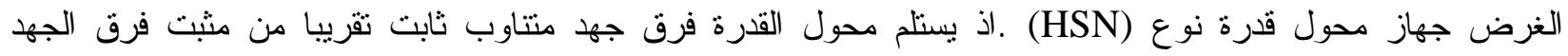
بمقدار (220V) ويزود فرق جه منتاوب بمدى (L-250V) ولغرض قياس الفولتية الخارجة استخدم جهاز رقمي منعدد

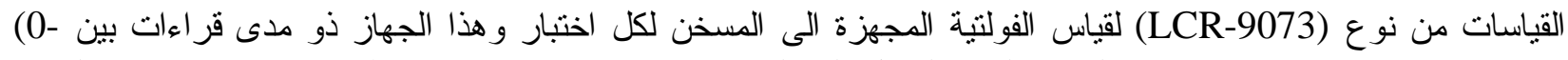

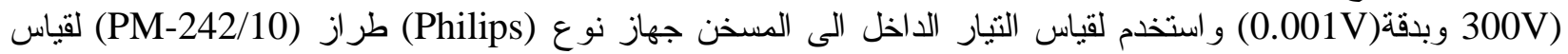

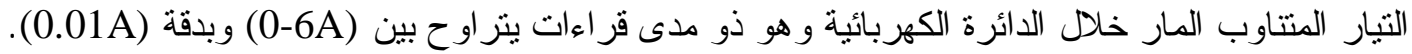

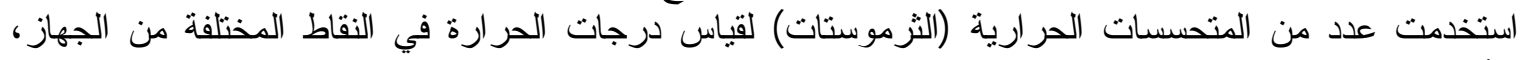

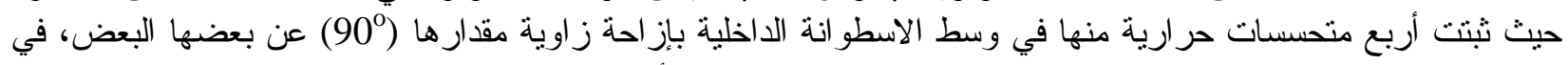

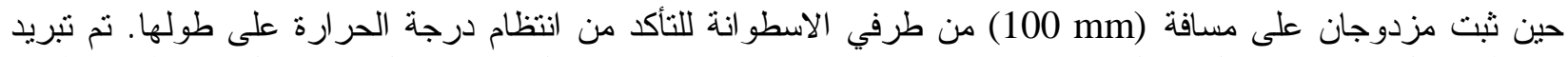

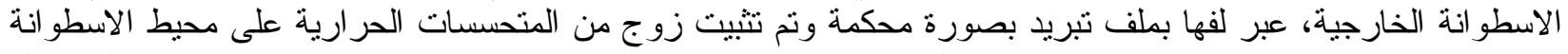

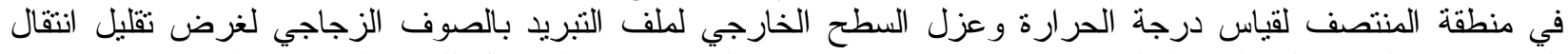

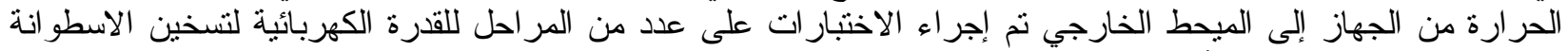

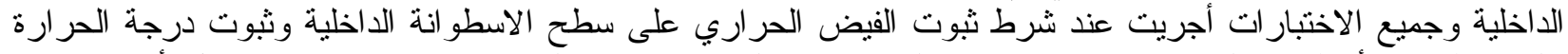

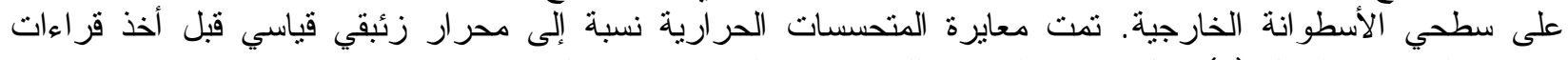

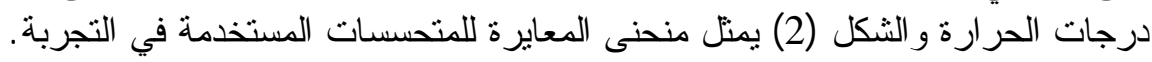

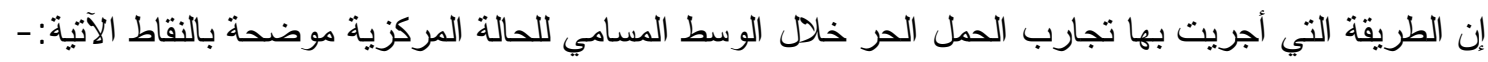

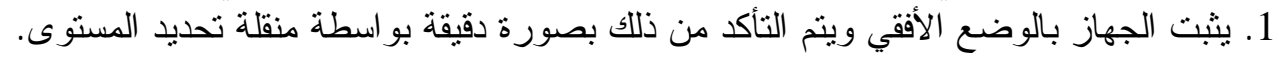

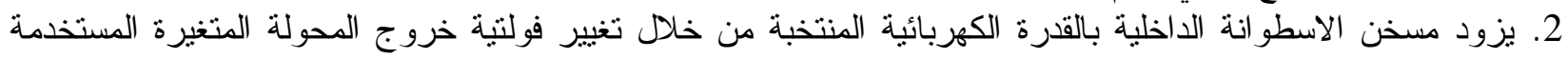

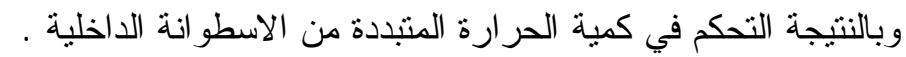

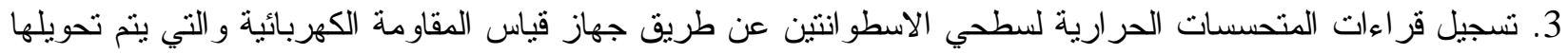

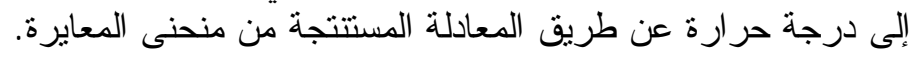
4. يتم الاستمر ار في اخذ القراء اته كل 10 دقائق حتى الوصول إلى حالة الاستقرار ، حيث يتم تسجيل قيم التيار و الفولتية المجززين للمسخن. 5. تغير القدرة المجهزة إلى المسخن، وتعاد الخطوات(4, 3) أعلاه في كل مرة. 


\section{Al-Rafidain Engineering $\quad$ Vol.17 $\quad$ No.6 $\quad$ Dec. 2009}

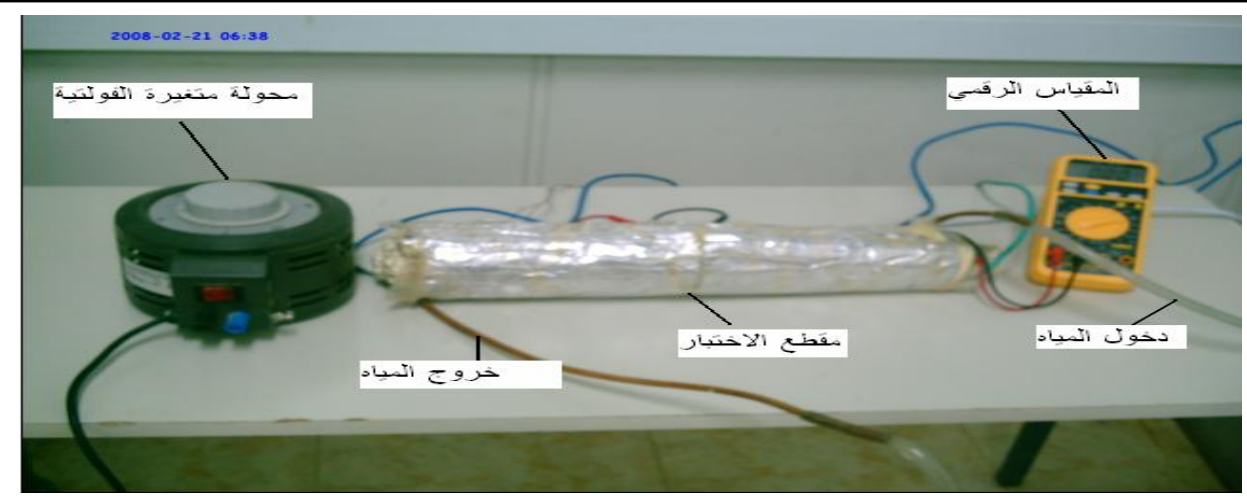

$$
\text { الشكل (1) المنشأ التجريبي المستخدم في البحث الحالي }
$$

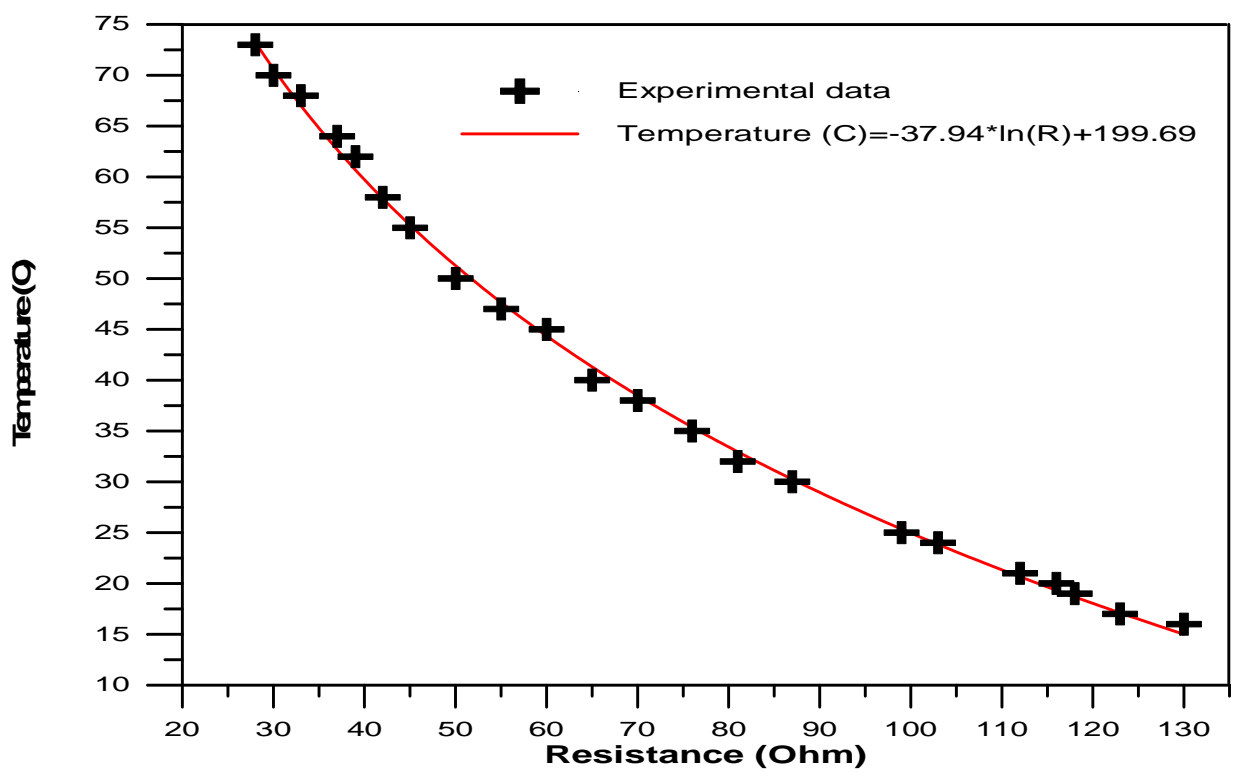

\section{الشكل (2) منحنى المعايرة للمتحسسات الحرارية (Thermistor) المستخدمة في التجربة}

لغرض وصف عملية انتقال الحرارة الخاصة بالدراسة الحالية نحتاج إلى قياسات وحسابات تتعلق بهذه العملية،

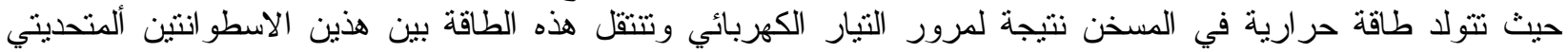

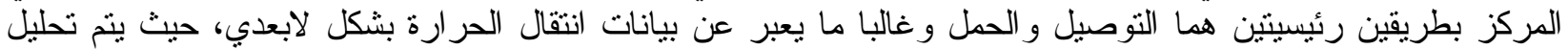

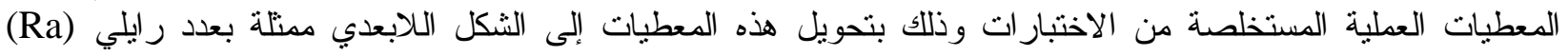
ومتوسط عدد نسلت (Nu) للتجارب العملية، تحسب القدرة الكلية المجزة للمسخن من المعادلة الآتية:

$Q=I^{*} V$

وبإهمال الخسائر الحرارية من الجوانب نتيجة العزل الجيد فان صافي معدل الحرارة المنتقلة بين الاسطو انتين سوف يكون مساوي إلى القدرة الكهربائية الداخلة وعلى هذا الأساس فان منوسط معامل انتقال الحرارة بالئ الحمل الحر تستخدم المعادلة

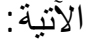

$$
\bar{h}=\frac{Q}{\pi d L \Delta T}
$$




\section{الجبوري : دراسة عملية ونظرية لاتقال الحرارة بالحمل الحر بين اسطوانتين متحدتي المركز مملوعة بوسط ...}

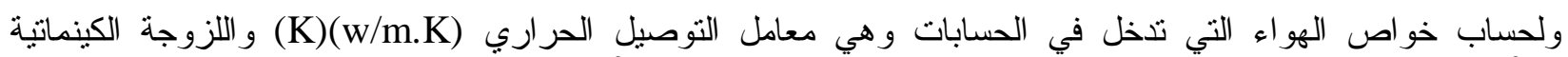
(v)(m²/s)

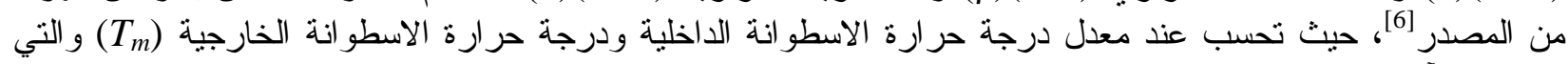
$T_{m}=\frac{T_{i}+T_{o}}{2}$ تحسب كالآتي :

\section{إما الإعداد اللابعدية اللازم حسابها فهي كالآتي: -}

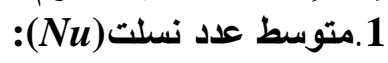

يمنل عدد نسلت معامل انتقال الحرارة اللابعدي، إذ يمنل نسبة الحمل الحراري إلى التوصيل الحر اري،ويحسب

$$
\text { متوسط عدد نسلت من المعادلة الآتية[7]: }
$$

حيث إن عeff معامل التوصيل الحراري الفعال ويحسب من المعادلة الاتية:

$$
k_{\text {eff }}=k_{f} \phi+(1-\phi) k_{s}
$$

2.

يعرف عدد رايلي على انه حاصل ضرب عدد كر اتشوف في عدد بر اندنل ويحسب من المعادلة الآتية[4]:

$$
R a=\frac{g \beta_{f} \rho_{o} \rho_{f} C_{p f}\left(R_{o}-R_{i}\right) \alpha\left(T_{i}-T_{o}\right)}{k_{e f f} \mu}
$$

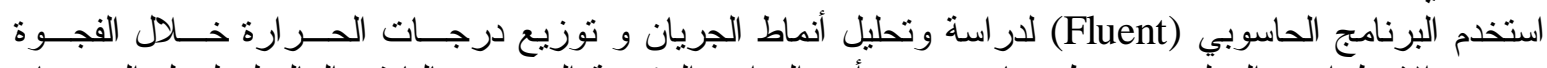

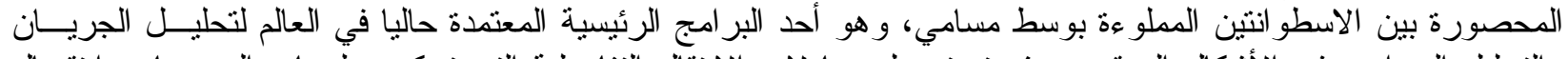

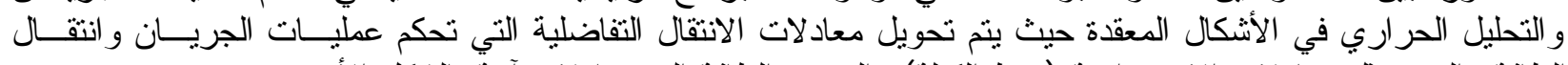

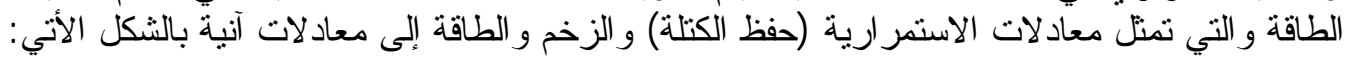
$\frac{\partial(\rho \phi)}{\partial t}+\frac{\partial(\rho u \phi)}{\partial x}+\frac{\partial(\rho v \phi)}{\partial y}+\frac{\partial(\rho w \phi)}{\partial z}=\frac{\partial}{\partial x}\left(\Gamma \frac{\partial \phi}{\partial x}\right)+$

$\frac{\partial}{\partial y}\left(\Gamma \frac{\partial \phi}{\partial y}\right)+\frac{\partial}{\partial z}\left(\Gamma \frac{\partial \phi}{\partial z}\right)+S$

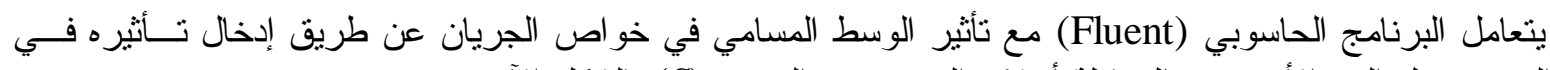

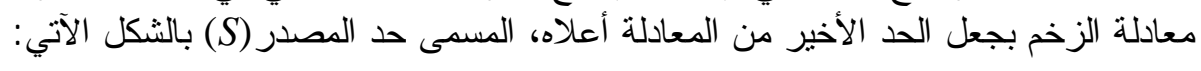
$S=-\left(\frac{\mu}{\mathrm{K}} v_{i}+C_{2} \frac{1}{2} \rho|v| v_{i}\right)$

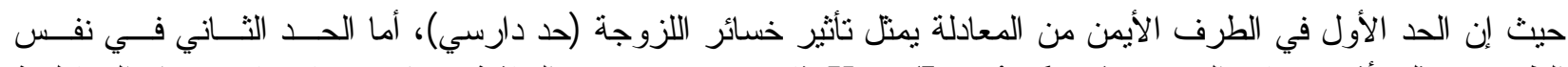

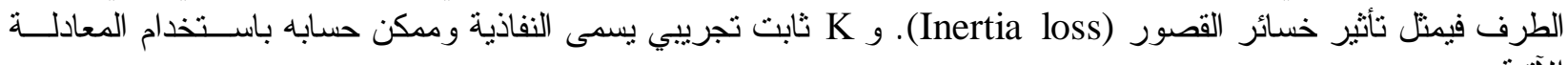


$\mathrm{K}=\frac{d_{p}^{2} \phi^{3}}{175(1-\phi)^{2}}$

حيث ان و انتل المسامية وتحسب من المعادلة الآتية:

$\varepsilon=\frac{V_{f}}{V_{t}}$

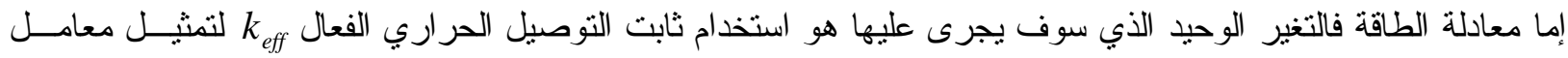

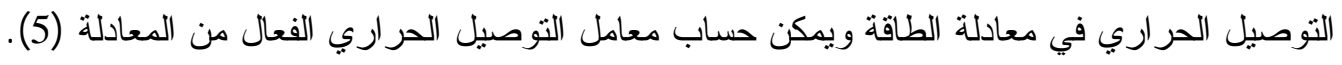

تحول معادلات الاستمر ارية و الزخم و الطاقة باستخدام طريقة الحجوم المحددة إلى معدلات آنية يمكن بو اسطتها إيجاد

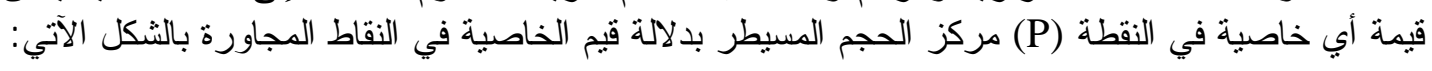
$a_{P} \phi_{P}=a_{W} \phi_{W}+a_{E} \phi_{E}+a_{S} \phi_{S}+a_{N} \phi_{N}+$

$a_{B} \phi_{B}+a_{T} \phi_{T}+a_{P}^{0} \phi_{P}^{0}+S_{u}$

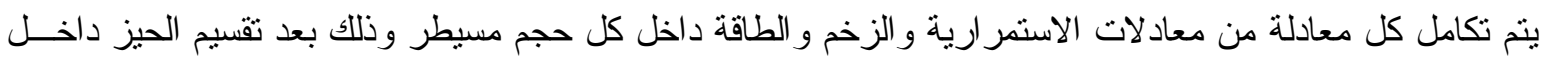

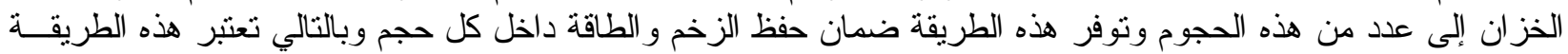

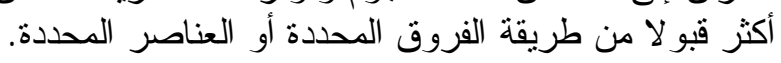

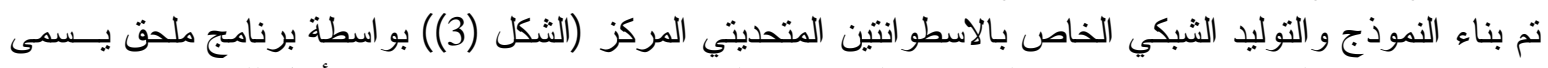

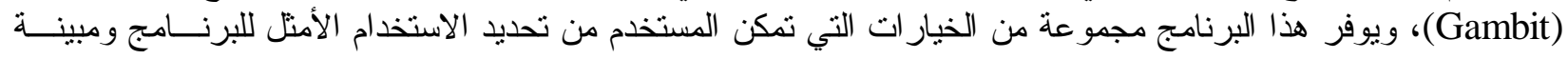

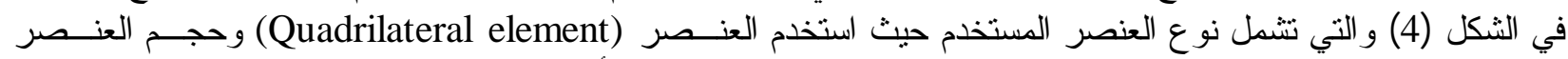

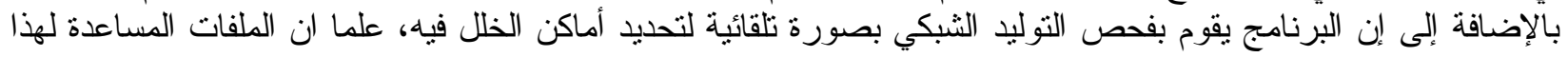

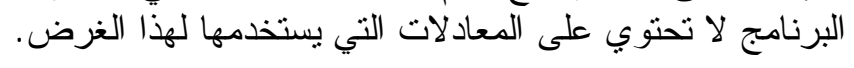

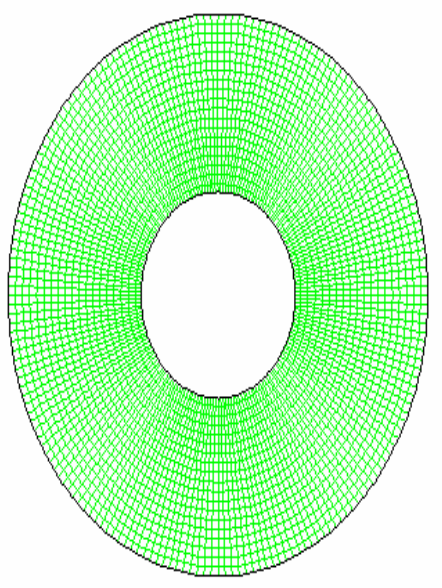

Girld
FLuENT 6.3 (2d, pbrs, , lamin)

الشكل (3) التوليد الشبكي للحيز المحصور بين

الاسطو انتين ألمملوء بوسط مسامي للميز المئ

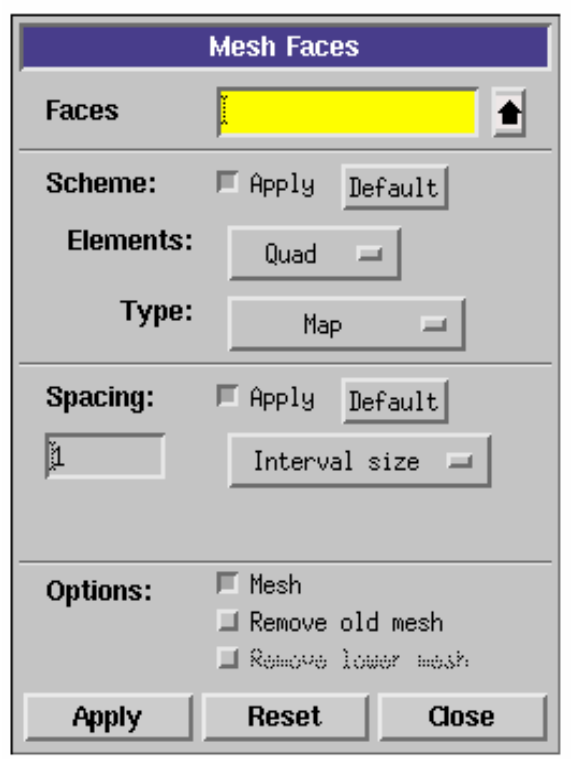

الثكل (4) واجهة البرنامج (Gambit) التي يتم فيها

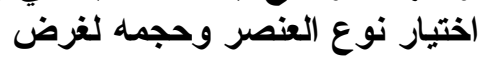

التوليد الثبكي للنموذج. 


\section{الجبوري : دراسة عملية ونظرية لاتقال الحرارة بالحمل الحر بين اسطوانتين متحتي المركز مملوءة بوسط ...}

تم استخدام نفس الظروف الحدية المستخدمة في الجانب العملي، وتم تسليطها وفق الخيار ات الموصى بها مــن قبــلـ الحسل

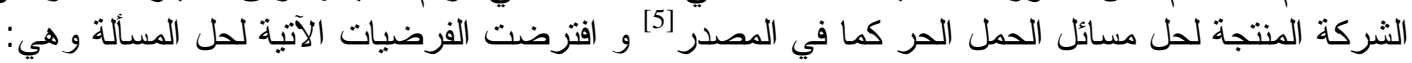

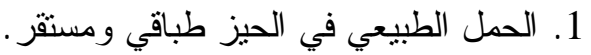

2. إهمال حد الانتشار (Viscous dissipation term) في معادلة الطاقة.

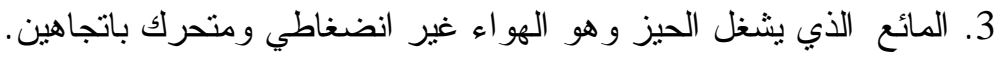

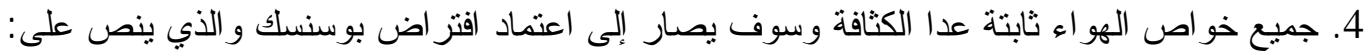

$$
\rho \cong \rho_{o} *\left(1+\beta *\left(T_{o}-T\right)\right)
$$

$$
\text { 6. اختيار الخو ارزمية (SIMPLE) لربط معادلات الزخم و الطاقة وهي ملائمة للحالات المستقرة. }
$$

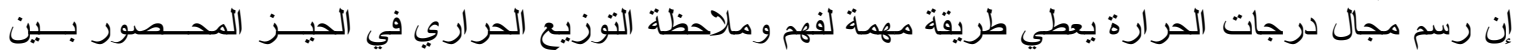

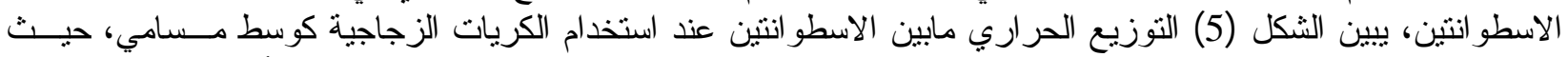

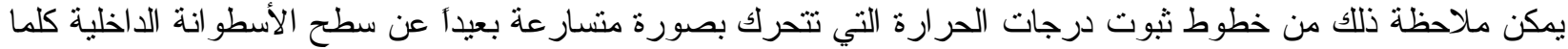

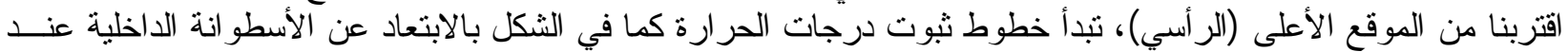

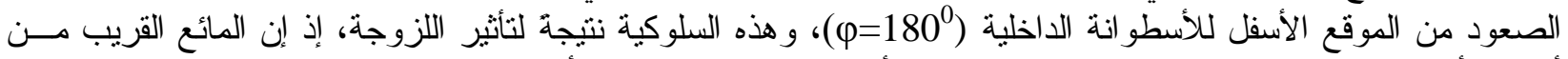

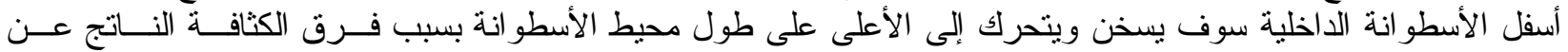

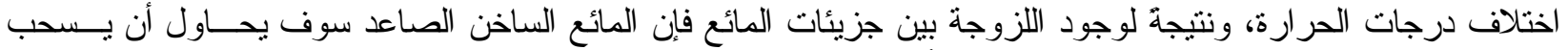

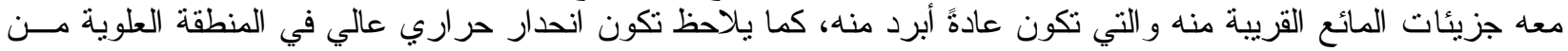

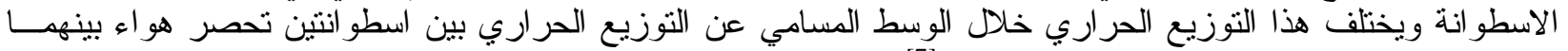

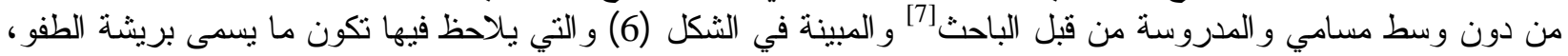

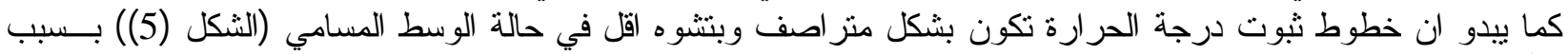

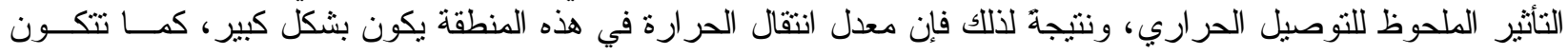

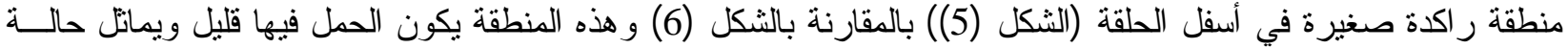

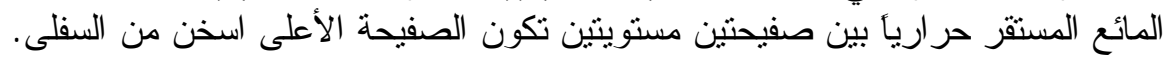

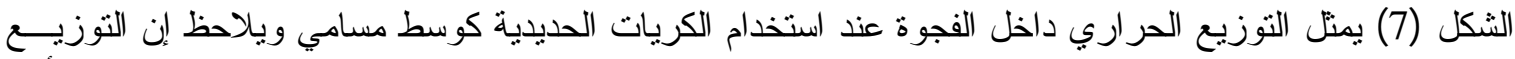

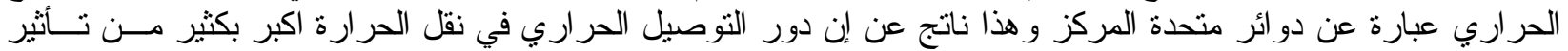

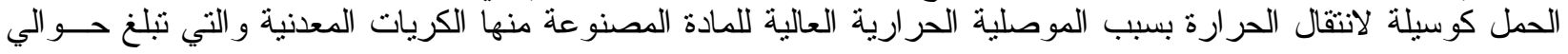
(15.6 W/m. C)

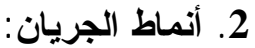

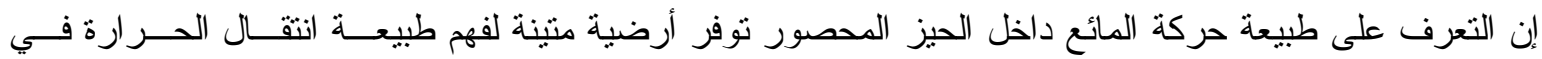

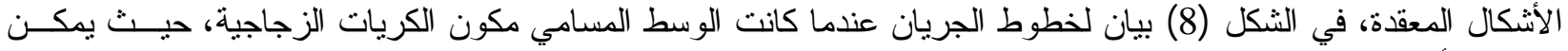

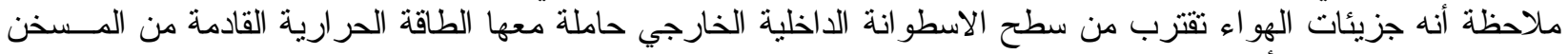

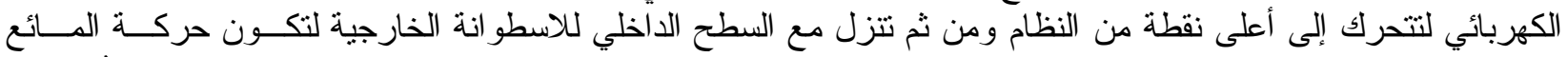

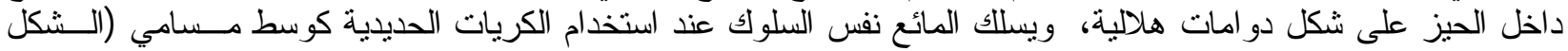

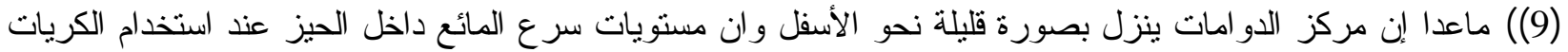

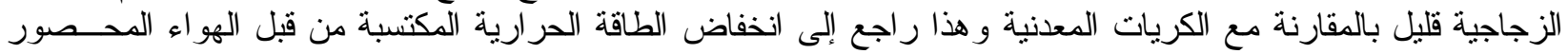

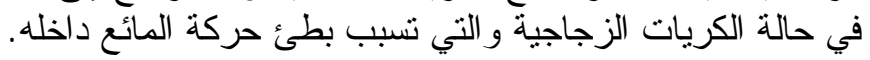




\section{Al-Rafidain Engineering}

Vol.17 No.6

Dec. 2009

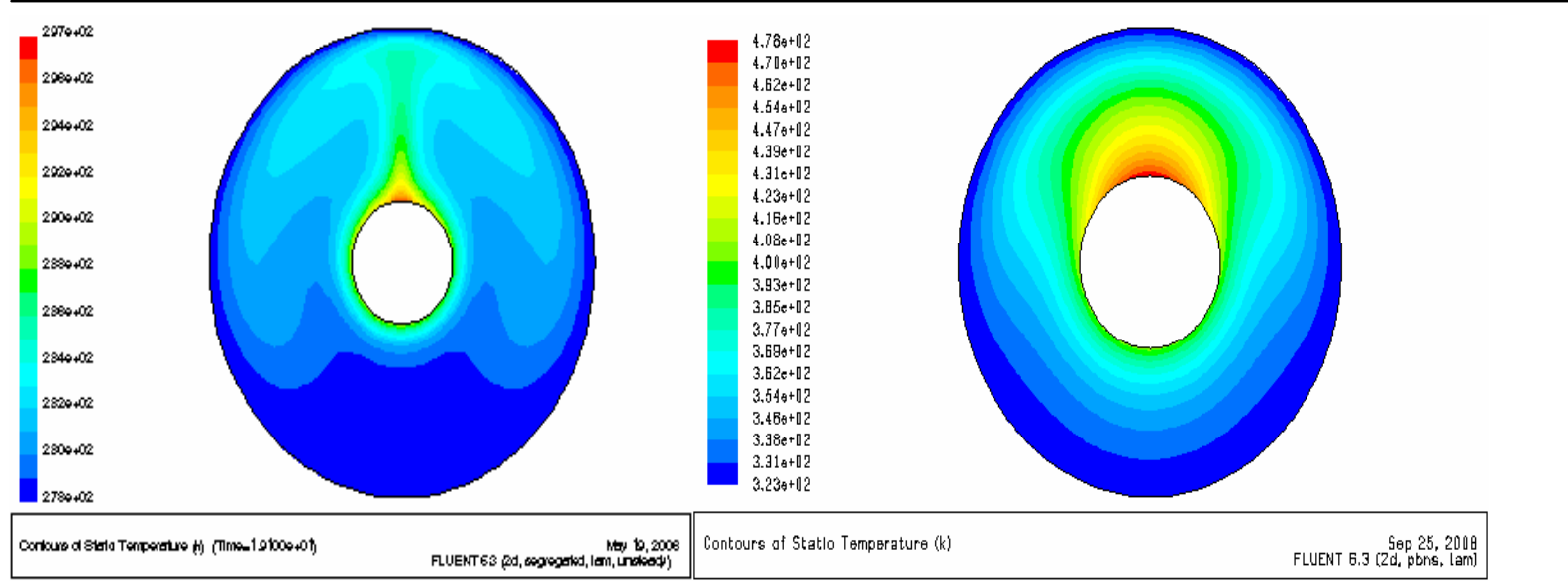

الشكل (5) التوزيع الحراري عندما كان الوسط الكيط

الثكل (6) التوزيع الحراري بين اسطوانتين

المسامي مكون من الكريات الزجاجية الوبطية

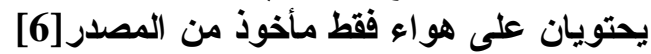
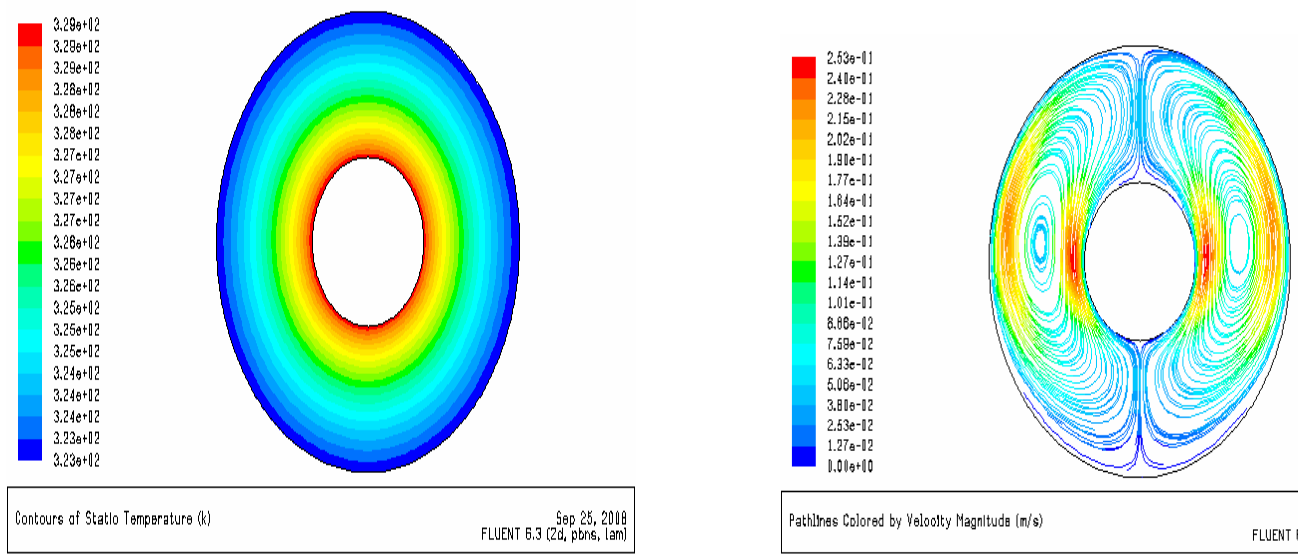

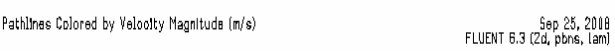

الثكل (7) التوزيع الحراري عندما كان الوسط المبط

المسامي مكون من الكريات المعدنية المئية

الثكل (8) أنماط الجريان للوسط المسام

لمكون من الكريات الزجاجية

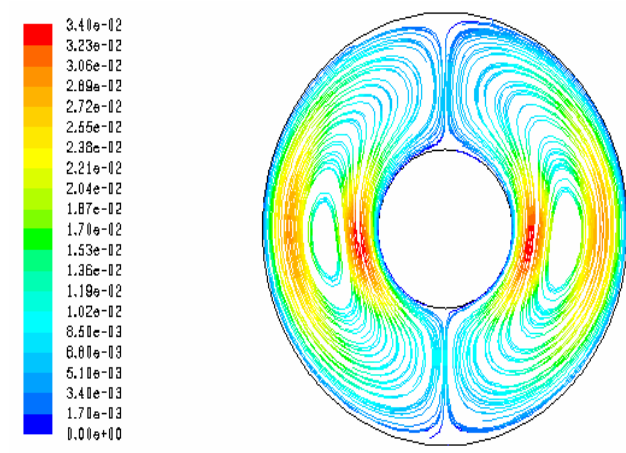

Pathlinas Colored by Yelootty Magnltuda (m/s)

$\operatorname{sep} 25,2000$
FLUENT 6.3 (2d, pbrs, lam)

الثكل (9) أنماط الجريان للوسط المسامي

لمكون من الكريات المعدنية 
3. تأثثر عدد رالي على التوزيع الحراري:

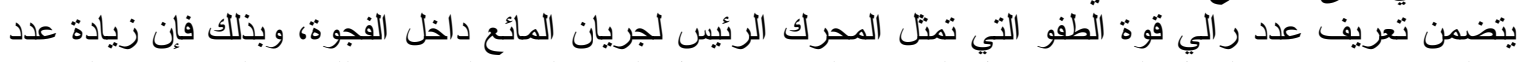

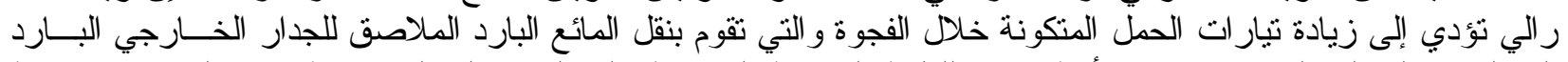

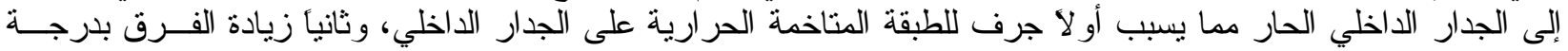

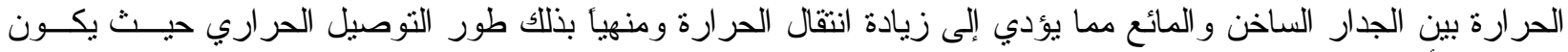

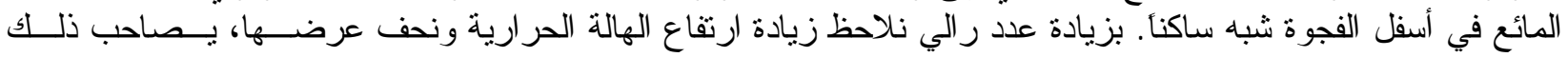
زيادة ملحوظة بانتقال الحرارة كما مبين في الثنكل (10).
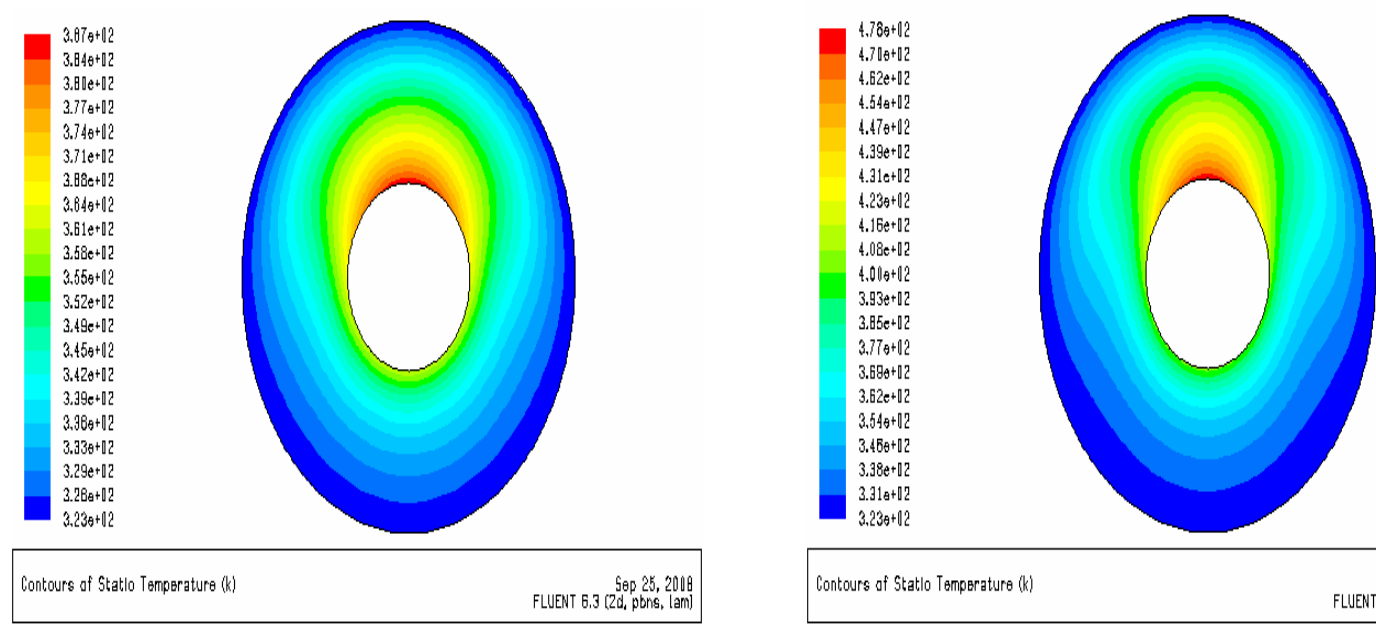

عدد ر ايلي = (160)

عدد ر ايلي = (313)

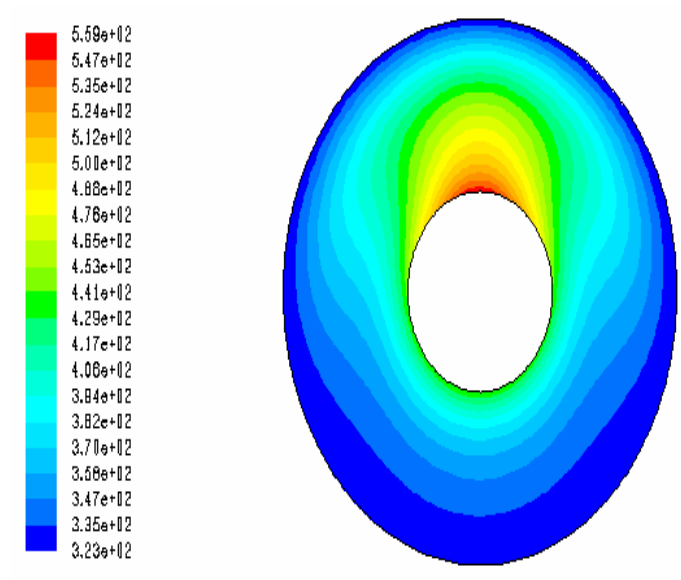

Contours of Statlo Temperature (k)

$5 \theta p 25,2000$
FLUENT $6.3(2 \mathrm{~d}, \mathrm{pbns}$, lam)

$$
\text { عدد رايلي = (590) }
$$

الشكل (10) تأثير عدد رالي على التوزيع الحراري 


\section{Al-Rafidain Engineering $\quad$ Vol.17 No.6 $\quad$ Dec. 2009}

4. المعادلات الارتباطية للنتائج العملية:

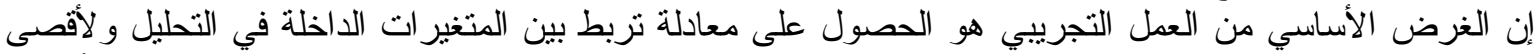

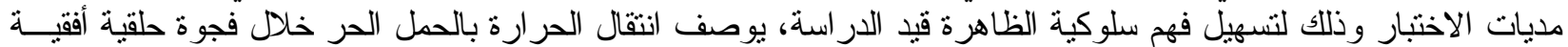

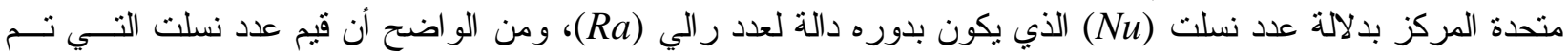
الحصول عليها من نتائج الدر اسة التجريبية ربطت بالمعادية بادلة الإرتباطية الآتية:

$N u=0.686+0.037 R a-0.000944(R a)^{2}+8.29349 \mathrm{E}-006(R a)^{3}$

حيث نلاحظ ان قيم عدد نسلت تزداد بزيادة عدد رالي ويمكن استخدام المعادلة أعلاه المستخلصة عملياً بنجاح في النتبؤ بالسلوك الحر اري للجريان خلال فجوة حلقية أفقية متحدة المركز مملوءة بودة بوسط مسامي بتأثير الحمل الحر وضمن أعداد

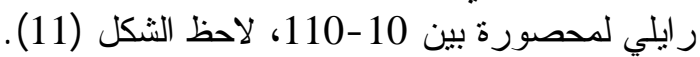

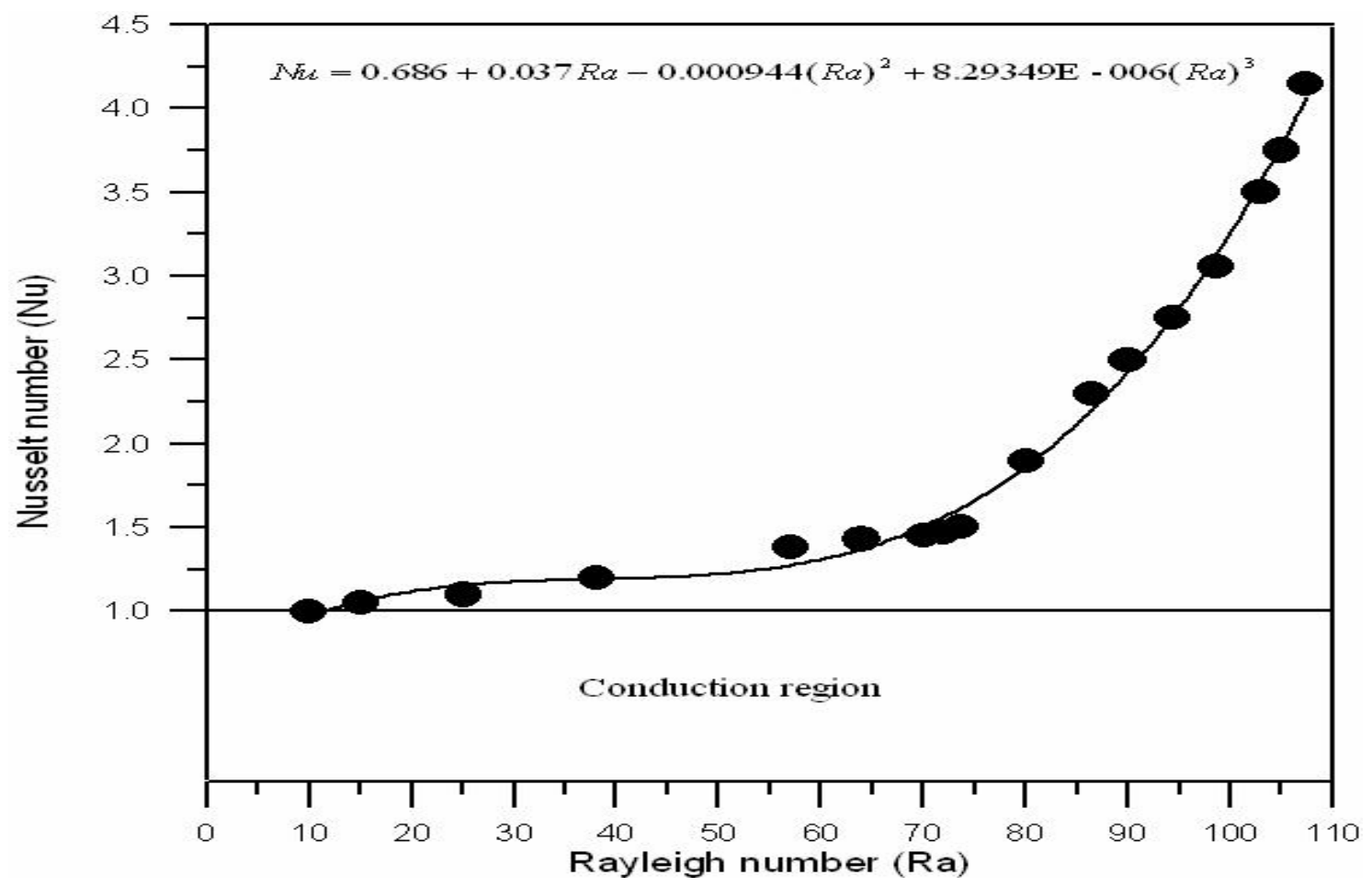

(11) الشكل (Nu) تغير عدد نسلت (Ra) مع عدد رايلي

الاستتتاجات و التوصيات:

من خلال النتائج المستحصلة واتوبات نجد ما يلي:

1. إن رسم مجال درجات الحر ارة يعطي طريقة مهمة لفهم وملاحظة التوزيع الحــراري فـــي الحيـز المحـصور بــين الاسطو انتين.

2. يختلف التوزيع الحراري في الوسط المسامي عن غير ها بكونه اقل حدة في الانحدار الحر اري.

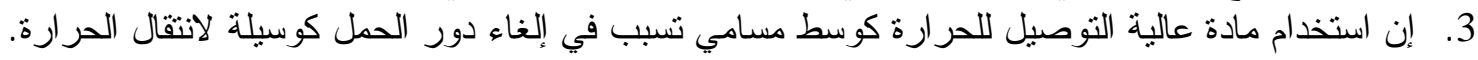

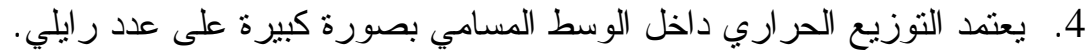

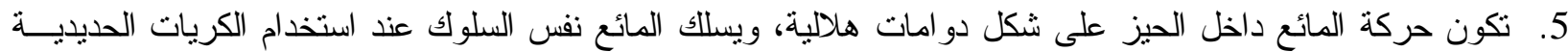

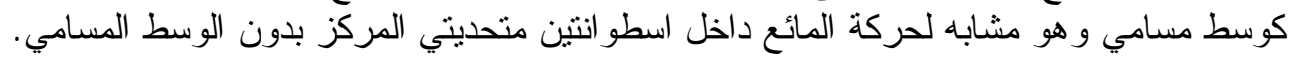

6. در اسة انتقال الحرارة بالحمل الحر بين اسطو انتي غير متحديتي المركز مملوعتين بوسط مسامي. 
1. المتيوتي، عطا له حسين جاسم، دراسة نظرية وعملية للحمل ألقسري الطباقي خلال أنبوب أفقي مملوء بوسط مسسامي مشبع. أطروحة ماجستير ، كلية الهندسة، جامعة تكريت.

2. Wang,B.X. and Zhang,X., Numerical and experimental investigations on the transient and steady-state natural convection in a vertical liquid saturated porous annulus. In heat transfer science and technology 1988, pp.417-423, Hemisphere, New york.

3. Wang,B.X. and Zhang,X., Transient natural convection in a horizontal liquid- saturated porous-media annulus. Chinese J. of engng thermophyse. Vol 10, no.3,pp 287-292, 1989.

4. Wang,B.X. and Zhang,X., Natural convection in liquid-saturated porous media between concentric inclined cylinders. Inter. J. of Heat and mass transfer, Vol 33, no.5, pp 827-833, 1990. 5. Braga ,E.J. and Lemos.J.S, Simulation of turbulent natural convection in a porous cylindrical annulus using a macroscopic two-equation model. Inter. J. of Heat and mass transfer, Vol 49, pp 4340-4351, 2006.

6. هرمز، جورج دانيال مطلوب ، تأثير الحاجز على انتقال الحرارة بالحمل الطبيعي في وسط مسـسامي بـين اســو انتين

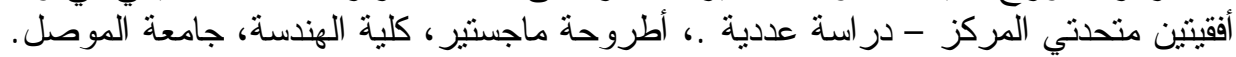

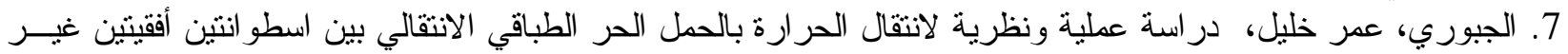
متحديتي المركز، مجلة جامعة تكريت للعلوم الهندية، المجلد (14)، العدد الأول،2007. 